\title{
Supporting Information for: Fundamental Limits on the Subthreshold Slope in Schottky Source/Drain Black Phosphorus Field-Effect Transistors
}

\author{
Nazila Haratipour, Seon Namgung, Sang-Hyun Oh, and Steven J. Koester* \\ Department of Electrical and Computer Engineering, University of Minnesota, \\ 200 Union Street SE, Minneapolis, Minnesota 55455, United States \\ * Address correspondence to: skoester@umn.edu
}




\section{Device Fabrication Process}

a


Figure S1. Fabrication sequence for BP MOSFETs. (a) $\mathrm{Si} / \mathrm{SiO}_{2}$ starting substrate, (b) local back gate formation, (c) $\mathrm{ALD} \mathrm{HfO}_{2}$ deposition, (d) black phosphorus alignment and transfer, (e) source and drain contact formation, (f) $\mathrm{ALD} \mathrm{Al}_{2} \mathrm{O}_{3}$ passivation layer deposition. 


\section{Optical Micrographs of Additional Devices}
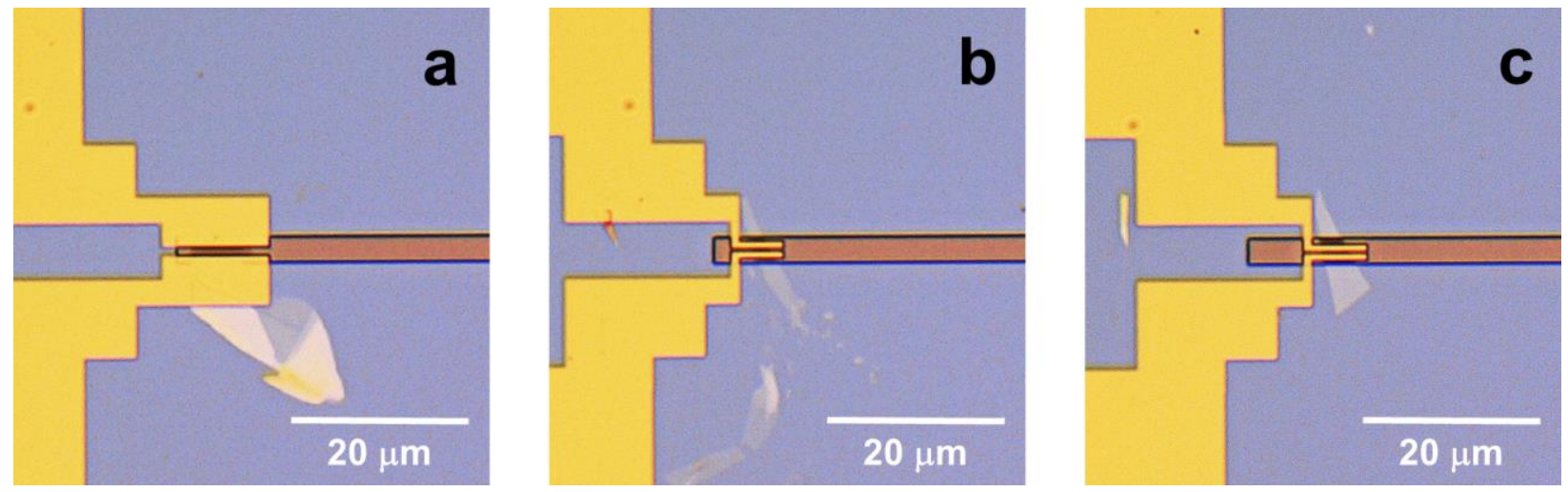

Figure S2. Optical micrographs of several completed BP MOSFETs. (a) Device \#2 with BP thickness (in the active device region), $t_{B P}$ of $4.3 \mathrm{~nm}$. The device width, $W_{g}$ was $7.15 \mu \mathrm{m}$ and the source-to-drain spacing, which defined the effective gate length, $L_{\text {eff }}$ was $1.0 \mu \mathrm{m}$. (b) Device \#3 with $t_{B P}=4.5 \mathrm{~nm}, W_{g}=2.00 \mu \mathrm{m}$ and $L_{e f f}=0.4 \mu \mathrm{m}$, and (c) Device \#7 with $t_{B P}=8.1 \mathrm{~nm}, W_{g}=$ $3.16 \mu \mathrm{m}$ and $L_{e f f}=0.30 \mu \mathrm{m}$. 


\section{Black Phosphorus Raman and Reflectance Characterization}
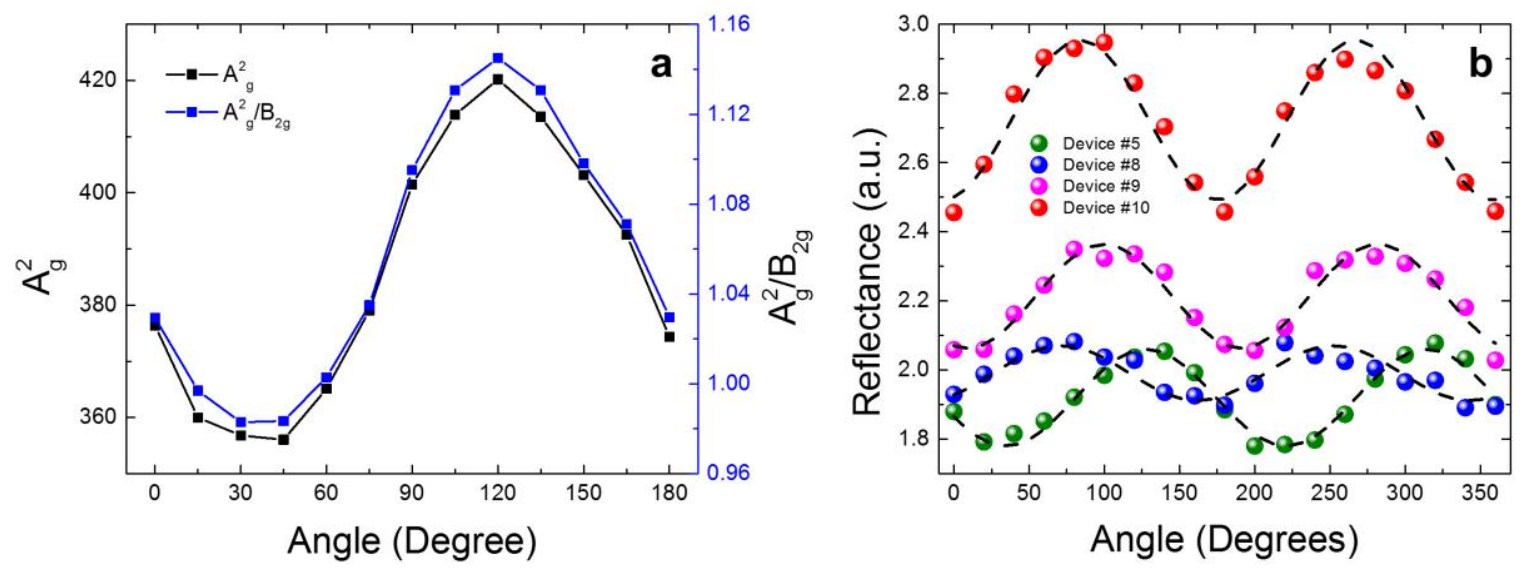

Figure S3. (a) Angular dependence of the Raman $\mathrm{A}_{\mathrm{g}}^{2}$ mode (left axis) and $\mathrm{A}_{\mathrm{g}}^{2} / \mathrm{B}_{2 \mathrm{~g}}$ (right axis) for a typical BP flake. The peak intensity is the direction that the laser polarization is along the armchair (low mass) direction. (b) Reflectance intensity as a function of rotational angle for devices $\# 5,8,9$, and 10. The maximum peak intensity indicates the zigzag direction (high mass). Dashed lines shows the sinusoidal fit of the reflectance data. 


\section{Double Sweep Transfer Characteristics}


Figure S4. Double sweep transfer characteristic of device \#3 (a) before and (b) after passivation for values of $V_{D S}=-0.1$ to $-2.5 \mathrm{~V}$ in $-0.2 \mathrm{~V}$ steps. Possible causes for the hysteresis include charge trapping in the $\mathrm{HfO}_{2}$ gate dielectric, moisture trapped underneath the $\mathrm{BP}$, and/or surface oxide layers on top of the BP. The hysteresis was greatly reduced after passivation, which we attribute to moisture desorption during the passivation layer deposition step at $200^{\circ} \mathrm{C}$. 


\section{Output and Transfer Characteristics}
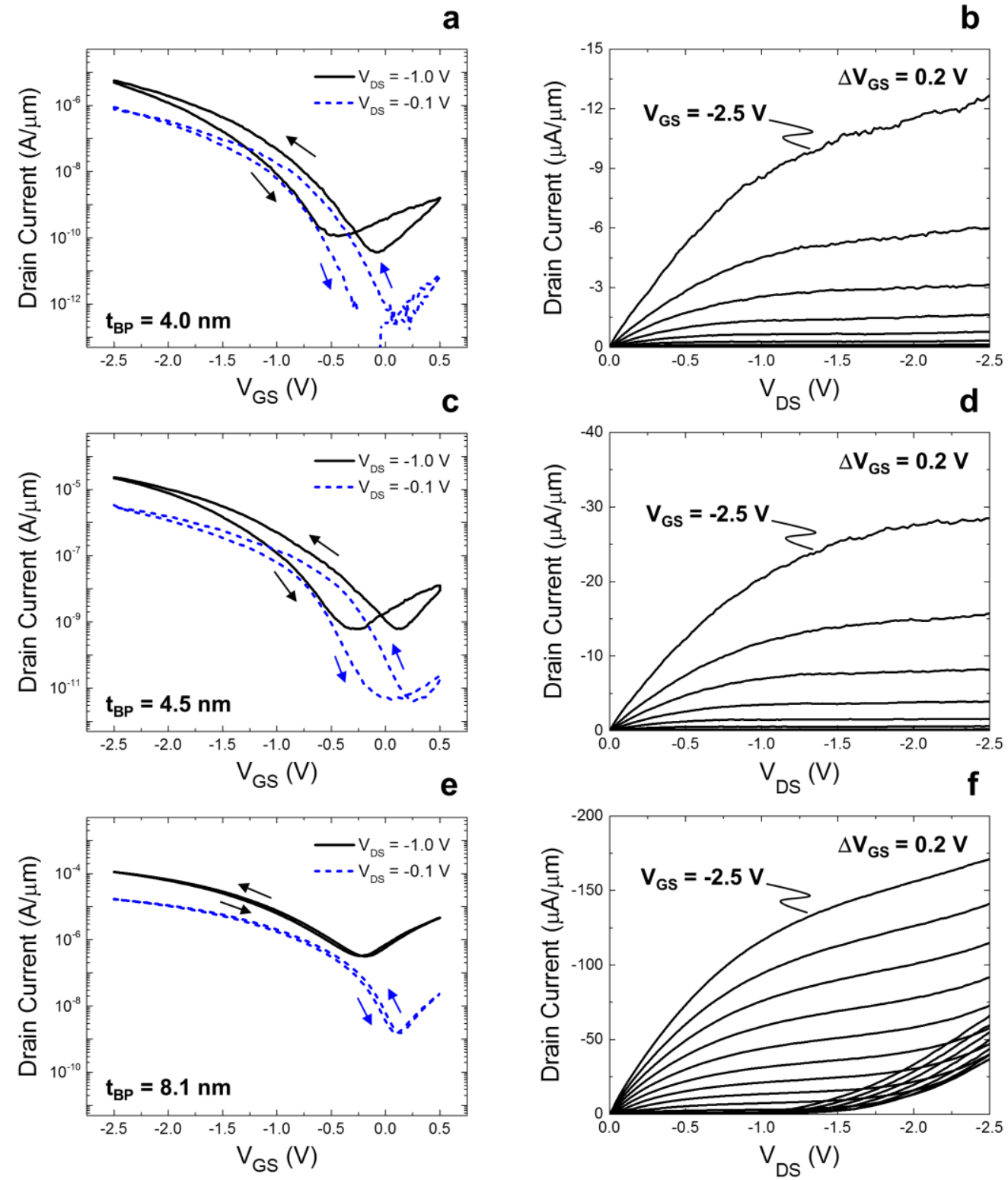

Figure S5. Room-temperature current-voltage characteristics of (a)-(b) device \#1 $\left(t_{B P}=4.0 \mathrm{~nm}\right)$ before passivation, (c)-(d) device \#3 $\left(t_{B P}=4.5 \mathrm{~nm}\right)$ before passivation, and (e)-(f) device \#7 ( $t_{B P}$ $=8.1 \mathrm{~nm}$ ), after passivation. (a), (c) and (e) are plots of drain current, $I_{D}$, vs. gate-to-source voltage, $V_{G S}$, for both forward and reverse sweep directions at drain-to-source voltage, $V_{D S}=$ $-0.1 \mathrm{~V}$ (blue) and $-1.0 \mathrm{~V}$ (black). (b), (d) and (f) show plots of $I_{D} v s . V_{D S}$ for the same devices where the top curves are at $V_{G S}=-2.5 \mathrm{~V}$ and the gate voltage step, $\Delta V_{G S}=+0.2 \mathrm{~V}$. 


\section{Black Phosphorus Stability Analysis}
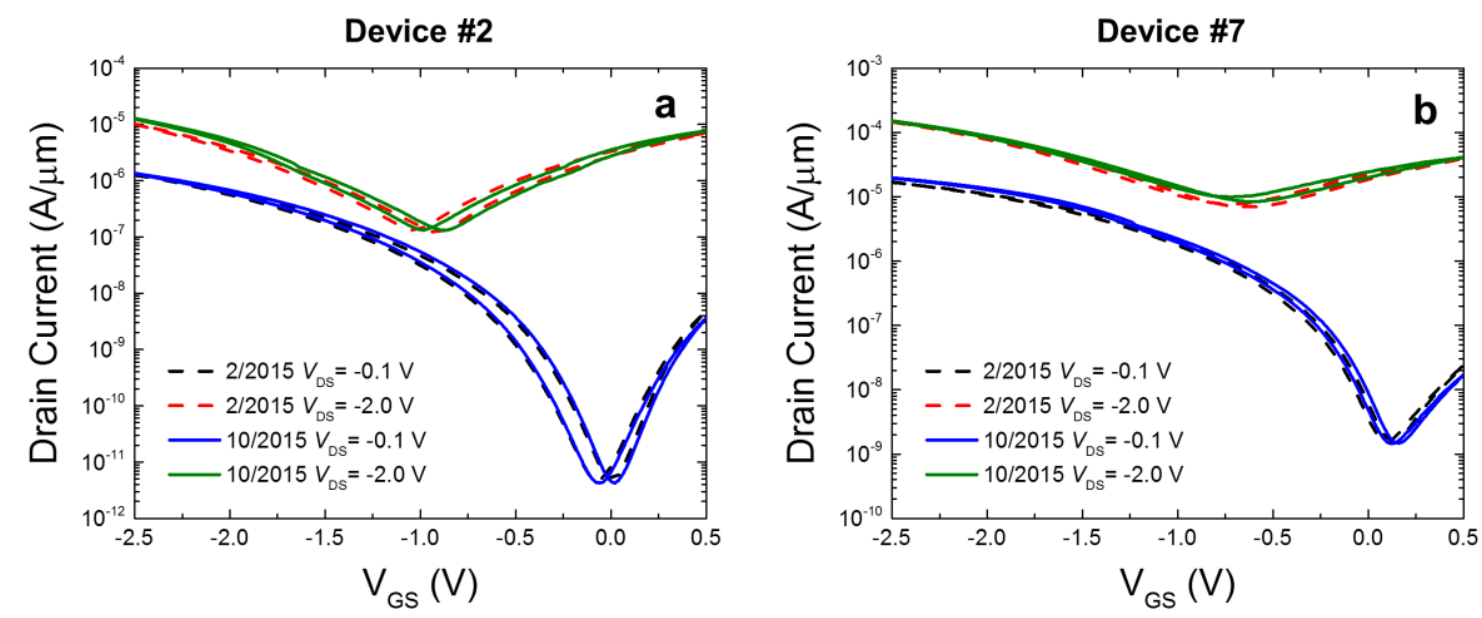

Figure S6. Comparison between the initial $I_{D} v s . V_{G S}$ characteristic of (a) device \#2 and (b) device \#7 immediately after passivation (in 2/2015) and the characteristics after $\sim 8$ months (in 10/2015). The devices were stored in a nitrogen purged dry box, but were exposed to air occasionally for characterization periodically during this time. Before the measurements shown on $10 / 2015$, the devices were first baked at $200{ }^{\circ} \mathrm{C}$ for 30 minutes. As shown in the figure, the threshold voltage and basic characteristics were completely retained, both at low and high values of $V_{D S}$. These results demonstrate the potential for long-term stability in black phosphorus MOSFETs passivated with $\mathrm{Al}_{2} \mathrm{O}_{3}$. 